\title{
Arquitectura e ingeniería en Alcoy. 90 años del puente de San Jorge
}

\section{Architecture and engineering in Alcoy. 90 years of the bridge of San Jorge}

\author{
J. F. Picó Silvestre ${ }^{(*)}$
}

\section{RESUMEN}

A finales del año 1925 se comenzó en Alcoy la construcción del puente de San Jorge. Se trata de uno de los primeros viaductos de grandes dimensiones construido enteramente en hormigón armado visto de España, pero además constituye uno de los únicos ejemplos contundentes que utilizó el lenguaje formal Art Déco en sus elementos. El 90 aniversario de esta construcción debe servir para conmemorar el éxito de una estrecha colaboración entre ingeniería y arquitectura. Este puente tuvo peculiares problemas y modificaciones importantes durante la construcción que fueron resueltos satisfactoriamente en un momento de la historia en el que la cultura se dirigía hacia la modernidad. En el caso que nos ocupa, el oficio del ingeniero y del arquitecto, oportunamente elegidos por una empresa constructora sagaz y efectiva en sus habilidades constructoras, dio como resultado una magnífica obra olvidada que conviene rehabilitar en la memoria colectiva.

Palabras clave: puente; hormigón; Alcoy; Art-Déco; Eusa; Monzón.

\begin{abstract}
At the end of 1925 the construction of Saint Jorge's bridge began in Alcoy. This was one of the first large viaduct built entirely of reinforced concrete in Spain, but it also constitutes one of the unique overwhelming examples that the language of Art Deco used in its elements. The 9oth anniversary of this bridge may serve to commemorate the success of a close collaboration between engineering and architecture. This viaduct had peculiar problems and important modifications during the construction that were resolved satisfactorily in a period of history where culture was heading towards modernity. In the present case, the work of the engineer and architect, duly selected by a shrewd and effective construction company in their building abilities, resulted in a magnificent forgotten structure that should be rehabilitated in the collective memory.
\end{abstract}

Keywords: bridge; concrete; Alcoy; Art-Déco; Eusa; Monzón.

(*) Departament de Composició Arquitectònica, Universitat Politècnica de València - Campus d'Alcoi (Alacant, España). Persona de contacto/Corresponding author: juapisil@cpa.upv.es (J. F. Picó Silvestre)

ORCID: http://orcid.org/oooo-0001-7063-2298 (J. F. Picó Silvestre)

Cómo citar este artículo/Citation: Picó Silvestre, J. F. (2016). Arquitectura e ingeniería en Alcoy. 90 años del puente de San Jorge. Informes de la Construcción, 69(545): e186, doi: http://dx.doi.org/10.3989/ic.15.146.

Copyright: (C) 2017 CSIC. Licencia / License: Salvo indicación contraria, todos los contenidos de la edición electrónica de Informes de la Construcción se distribuyen bajo una licencia de uso y distribución Creative Commons Attribution License (CC BY) Spain 3.o. 


\section{INTRODUCCIÓN}

Frente al concepto estricto de puente, la palabra viaducto enriquece el lenguaje añadiendo la idea de la conducción de un camino, de una vía sobre un accidente topográfico continuándola hacia su destino. Si además este camino se convierte en una calle y su existencia resulta esencial para el desarrollo de una ciudad, la intensidad de su historia crece junto a la de sus ciudadanos. A finales de noviembre de 1925, hace noventa años, se inició la construcción del puente de San Jorge en Alcoy (Alicante). Fue uno de los primeros puentes construidos en hormigón visto de España, pero además de sus avanzados valores tecnológicos y urbanísticos, constituye uno de los pocos ejemplos mundiales de la ingeniería civil con un lenguaje formal Art Déco.
Este puente absolutamente urbano tiene una composición particular fruto de la propia historia de su proyecto y de su ejecución. La parte original tiene 156 metros de longitud y está constituida por tres vanos con arcos parabólicos de 46 metros, contando con dos pilas centrales. Tiene una altura de hasta 45 metros y un tablero horizontal con un ancho total de 12 metros. Pero este tramo viene completado en el lado Norte por un tablero inclinado $(3,62 \%)$, con cuatro vanos de casi 19 metros apoyados sobre pilastras. Así pues la longitud total del puente es de 251 metros.

El proyecto de este viaducto se realizó para acortar distancias entre el centro de la ciudad y las estaciones de los ferrocarriles de Alcoy, pero sobre todo para hacer más accesible la zona del ensanche de la ciudad con objeto de ofrecer suelo más

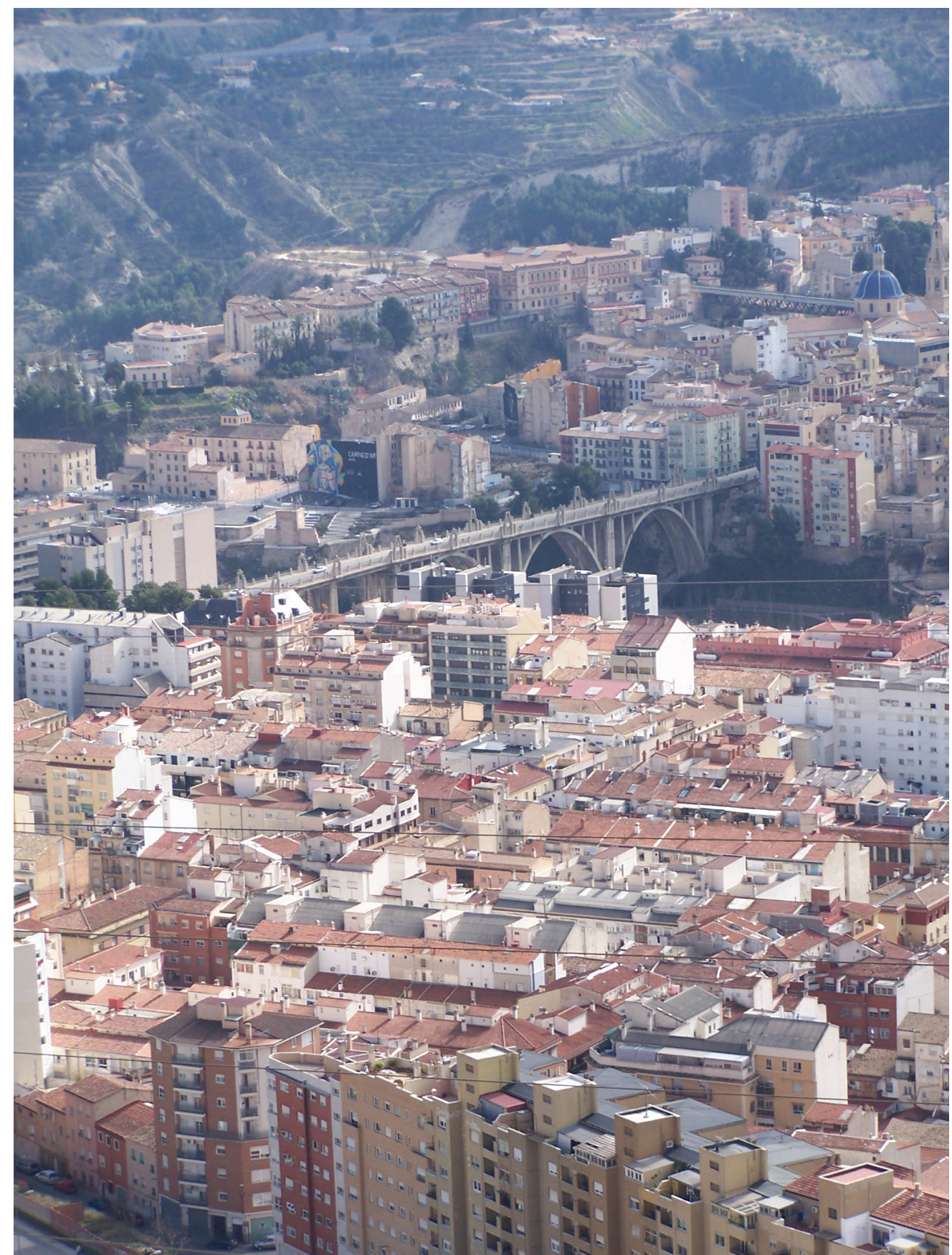

Figura 1. Inserción urbana del puente (fotografía de la colección del autor). 
cercano al centro y así paliar la escasez de vivienda que se ofrecía entonces en el casco urbano (Figura 1).

El proyecto y la construcción de este viaducto estuvo bajo la dirección de los ingenieros de caminos Carmelo Monzón y Repáraz y de Vicente Redón Tapiz. No obstante, el resultado formal del puente corresponde decididamente a la participación del arquitecto Víctor Eusa Razquín en el proyecto. La empresa que construyó el puente fue Erroz y San Martín de Pamplona y actuó como ingeniero supervisor de la obra por parte de la administración Alfonso Peña Boeuf (1). La obra finalizó el 1 de julio de 1927, pero el puente no se inauguró ni entró en funcionamiento hasta el 26 de marzo de 1931.

\section{INGENIERÍA Y ARQUITECTURA. UNA RELACIÓN FRUCTÍFERA}

En general, la relación entre los ingenieros y los arquitectos no siempre fue cómoda en la construcción. Sin embargo, el caso del puente de San Jorge de Alcoy es un ejemplo de éxito a causa de las circunstancias y disposiciones personales de los jóvenes y brillantes Carmelo Monzón, ingeniero, y Víctor Eusa, arquitecto.

En la construcción de puentes de hormigón armado en España la colaboración entre ingenieros y arquitectos surge casi al mismo tiempo que el propio uso de este material. El hormigón armado aparece en España a finales del siglo xIX asociado a los procedimientos constructivos y a soluciones estructurales para obras civiles singulares (depósitos, silos, etc.). Estos sistemas constructivos están patentados por firmas interna- cionales como Monier o Hennebique y de inmediato serán replicados por empresas españolas dirigidas por ingenieros (2).

Uno de los primeros ejemplos documentados de la colaboración entre arquitectura e ingeniería es el caso del concurso para el puente de María Cristina de San Sebastián (19031905), en cuyas bases se justifican ampliamente las razones para este tipo de colaboración. Por un lado, parece que el ingeniero es capaz de lograr el equilibrio justo entre la utilidad, la estabilidad y la economía. La utilitas y la firmitas de Vitrubio con el filtro de la economía, quizás para someter las veleidades de la belleza (venustas). Un puente se considera una obra monumental, un monumento que necesariamente implica grandiosidad en su aspecto y riqueza en los detalles, atributos que Palladio confería a los edificios públicos y obras civiles. Por el otro lado, el arte de los arquitectos podría aportar la elegancia a este equilibrio y así conseguir «ese sentimiento vago y confuso que despierta en el alma el culto a lo bello» (3).

José Eugenio Ribera, ingeniero ganador de este concurso, unos años después al final de una conferencia, defendía la necesidad de dar carácter monumental a los puentes a través de la intervención de los arquitectos, puesto que «en general se proyectaban con cierta mezquindad» (4). Más adelante, Ribera ajustará sus criterios de gusto sobre el uso del hormigón armado en los edificios con ocasión de la British Empire Exibition de 1924 en Londres. En la visita a esta exposición sufrió una cierta decepción artística. En su crónica estableció el debate entre el excesivo recurso al manoseado clasicismo en muchos de los edificios y el inicio de un nuevo

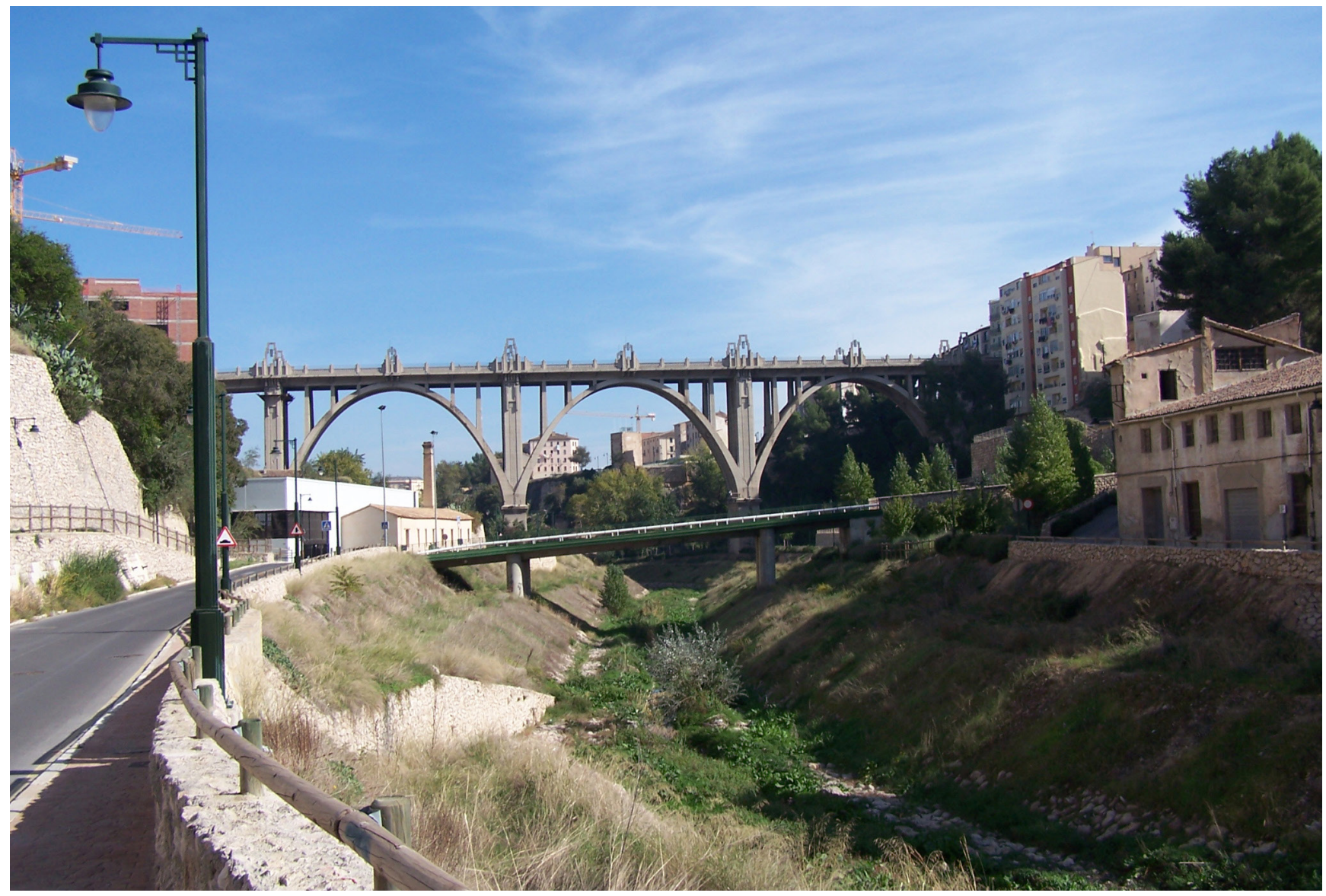

Figura 2. Vista del tramo de los arcos del puente desde aguas arriba (fotografía de la colección del autor). 
estilo que expresaba la sinceridad constructiva de los grandes palacios construidos con hormigón, con un aspecto gris, frío y monótono. Aplaudía la sinceridad constructiva, la desnudez, solamente si las formas eran perfectas de lo contrario se debía seguir decorando con discreción y ornamentando con mesura las construcciones de hormigón. Sólo las estructuras esenciales habrían de acusar sin falsedades la forma de toda la obra (5).

La trayectoria de Sir Owen Williams, responsable del proyecto y de la construcción de los edificios más importantes de esta exposición de Londres, expresa ejemplarmente la simbiosis de las dos disciplinas profesionales. Williams se tituló como ingeniero en 1911 y posteriormente se inscribió como arquitecto en 1930. Al principio no fue considerado un ingeniero-arquitecto perteneciente a la Modernidad sobre todo por su intervención en la British Empire Exibition, sin embargo, a partir de su gran capacidad y de su desarrollo en el dominio del hormigón armado, a través del empeño en construir con precisión y con rigor, con la búsqueda de la simplicidad y el gusto por las cosas bien hechas -actitudes tanto técnicas como estéticas- contribuyó al desarrollo de la arquitectura moderna en Inglaterra. Edificios como el Daily Express de Londres construido entre 1929-1931 con una singular estructura de hormigón armado, con un radical muro cortina en las fachadas y con un vestíbulo decorado con todas las características compositivas del Art Déco, constituyen un manifiesto de la Modernidad. Así, la importancia del detalle como material del proyecto y la actuación de síntesis del arquitecto sobre el trabajo del ingeniero capaz de moverse en cualquier escala, conocedor de los mecanismos de la depuración formal y de la abstracción a través de la experiencia tecnológica, asentaron las bases de la Modernidad (6).

Pues bien, la necesidad de sumar las concepciones del Arte a los procedimientos de la Ciencia fue una preocupación moderna para acercar la producción industrial y la estética de la máquina a la arquitectura. El uso de los beneficios de la formación Beaux Arts conjugados con los preceptos de L'École Polytechnique y la aceptación de las Vanguardias dieron como resultado el fenómeno estético del Art Déco. Este fenómeno artístico de la cultura occidental se manifestó principalmente durante el período transcurrido entre las dos guerras mundiales del siglo xx. Tuvo una gran repercusión en la Arquitectura y el Diseño y también contribuyó a modificar los estilos de vida y el modo de entender la cultura de la sociedad. En el momento de la concepción del puente de San Jorge para Alcoy éste es el debate propio de la Modernidad frente al agotamiento de las posiciones estéticas del siglo XIX. Uno de los hitos históricos importantes del Art Déco fue la Exposición Universal de las Artes Decorativas e Industriales Modernas de París celebrada entre los meses de abril y octubre de 1925, unos meses antes del inicio de la construcción del puente de San Jorge.

\section{LA EMPRESA CONSTRUCTORA}

El 8 de julio de 1923 el Ayuntamiento de Alcoy acordó encargar el proyecto y la construcción del puente a la empresa constructora Erroz y San Martín de Pamplona, que entonces trabajaba en Alcoy en la construcción de las Escuelas Industriales. El azar quiso que esta empresa estuviera presente en Alcoy con una obra de estas características. Sus orígenes parten del año 1890 a través de la construcción del ferrocarril del Irati en Navarra. Entre sus obras destacadas están la sede del Banco de España en Navarra, a la que años después siguió la construcción de una treintena de sedes para el Banco de España en otras ciudades, incluso la ampliación de la sede central de Madrid. En Valencia construyeron entre 1927 y 1928 el edificio de la Compañía Telefónica Nacional de España de la plaza del Ayuntamiento, bajo proyecto de los arquitectos Santiago Esteban de Mora e Ignacio de Cárdenas Pastor, redactado en 1926. En la Exposición Iberoamericana de Sevilla de 1929 construyeron el Pabellón de la Marina Mercante. Pero la obra que probablemente más nos interesa es la construcción durante el año 1924 del edificio de la compañía de Seguros

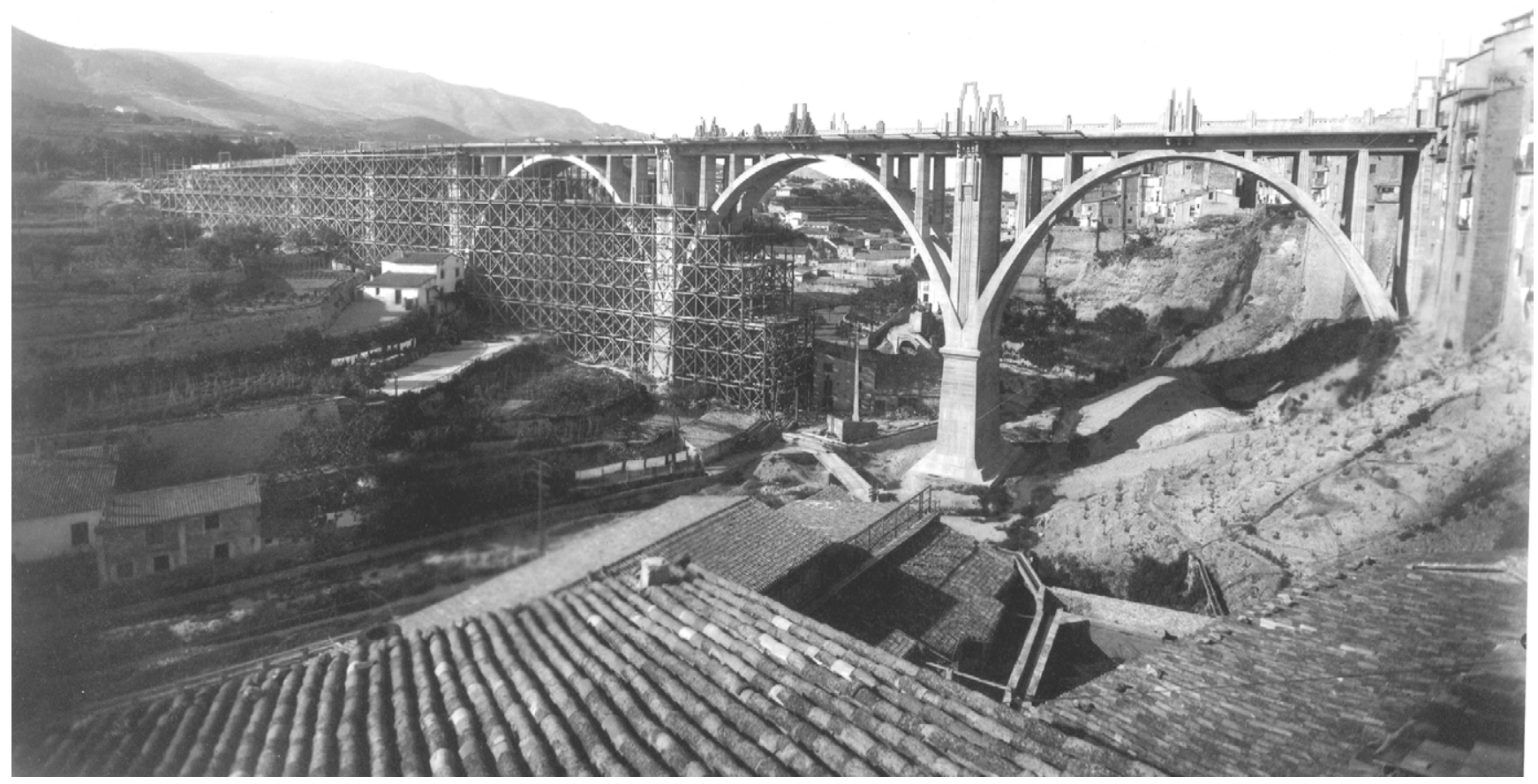

Figura 3. Puente de San Jorge con su cimbra en proceso de desmonte (fotografía del Archivo Municipal de Alcoy). 
La Vasco-Navarra de Pamplona, proyectado por Víctor Eusa, puesto que es la obra coetánea con la construcción del puente de San Jorge. La empresa Erroz y San Martín tenía como raíz fundamental la carpintería de cierta escala. Esto significa que, con toda probabilidad, disponían de carpinteros, de encargados propios altamente cualificados y adaptados a una técnica que exigía mucha competitividad en el reciente mercado de la construcción del hormigón armado.

\section{LOS INGENIEROS}

Erroz y San Martín, como empresa adjudicataria de las obras del futuro puente de San Jorge, debía de extraer ventajas de su posición e intentó, a buen seguro, encontrar algunos ingenieros de su entorno que conociera y con los que pudiera tener una excelente relación. Así planteó el proyecto a dos ingenieros pamploneses, jóvenes de excelente expediente académico, recién titulados en la Escuela de Ingenieros de Caminos de Madrid, bien conocidos por los ingenieros de la Administración Pública del Estado, muchos de ellos profesores de tal Escuela, y además procedentes del seno de familias reconocidas en la sociedad navarra: los ingenieros Carmelo Monzón Repáraz y Vicente Redón Tapiz. De Vicente Redón solamente hay referencias de su presencia durante la redacción del proyecto inicial, pero posteriormente no hay rastro de su presencia y todo el protagonismo documental recae sobre Carmelo Monzón.

Con respecto a su proyecto del Puente de San Jorge se cita lo siguiente: «...proyecto redactado por nuestro joven e inteligente compañero D. Carmelo Monzón, que estudió este viaducto con todo el entusiasmo de la primera obra y con toda la competencia de una sólida preparación» (7). Por lo tanto, su primer proyecto importante fue este puente para Alcoy. También se sabe que fue el número uno de su promoción de ingenieros de la Escuela de Madrid.

Con posterioridad a estas obras y en el período de la Guerra Civil española, Carmelo Monzón fue nombrado Inspector General de Obras Públicas del Gobierno Vasco de la II República mediante el decreto firmado por J. Astigarrabia, Consejero de Obras Públicas, el 4 de enero de 1937. Bilbao todavía no había sido tomada por las tropas franquistas. Fue confirmado en su puesto y ampliado sus funciones a todo el departamento en una remodelación habida el primero de abril de
1937. Dado que ejercía un puesto en la Administración del Estado de la República es muy posible que fuera represaliado de algún modo. También aquí conviene recordar el contexto en el que se movía este ingeniero. Precisamente, el 18 de julio de 1936, el general Emilio Mola produce uno de los focos de la sublevación del «alzamiento nacional» en la ciudad de Pamplona, después de una gestación largamente proyectada. Poco antes de la sublevación, Jesús Monzón Repáraz, hermano pequeño de Carmelo, crea la organización del Partido Comunista de España en Navarra. Los Monzón-Repáraz son «una de las mejores familias de Pamplona, hijo menor de un distinguido médico burgués y descendiente, por parte de madre, de un antiguo y blasonado linaje de la aristocracia rural de Navarra... cuando en Pamplona triunfa el golpe de Estado al que las amigas de su madre llevan años enteros rezándole novenas, y consigue escapar...» (8). Por lo tanto, Carmelo Monzón Repáraz, a pesar de pertenecer a una familia tradicionalista y afín a la ideología de los sublevados, tiene un hermano que fue un personaje muy conocido e importante en la superestructura del Partido Comunista de España en el exilio, que organizó la resistencia en el Sur de Francia y que durante algún tiempo fue uno de los pilares de la Secretaría General del Partido.

Es muy probable que Carmelo Monzón quedara en cierto modo deshabilitado como consecuencia de estas circunstancias políticas colaterales puesto que no hay más noticias suyas hasta algunos años después. Trabajó de ingeniero calculista de la estructura de hormigón en el proyecto del Edificio España de Madrid, proyectado en 1947 por Joaquín y Julián Otamendi Machimbarrena, y en el edificio Torre de Madrid en el año 1954 de los mismos arquitectos. Ésta era una actividad que seguramente ya había desarrollado en Pamplona con otros arquitectos como Víctor Eusa. Posteriormente, en 1951 fundó la empresa Sociedad Anónima de Hormigones Especiales (SAHE) que se dedicaba a fabricar elementos pretensados de hormigón, llegando a limitar su producción a la fabricación de viguetas de cemento aluminoso durante los años sesenta. Estos elementos de gran resistencia han tenido una fuerte repercusión social en la actualidad con la aparición de la patología de la "Aluminosis» y su uso dejó de estar permitido en 1980. La empresa SAHE distribuyó catálogos de sus productos entre los arquitectos alcanzando gran popularidad en el sector. La empresa desapareció en 1971. Carmelo Monzón también fue presidente del Consejo de Administración

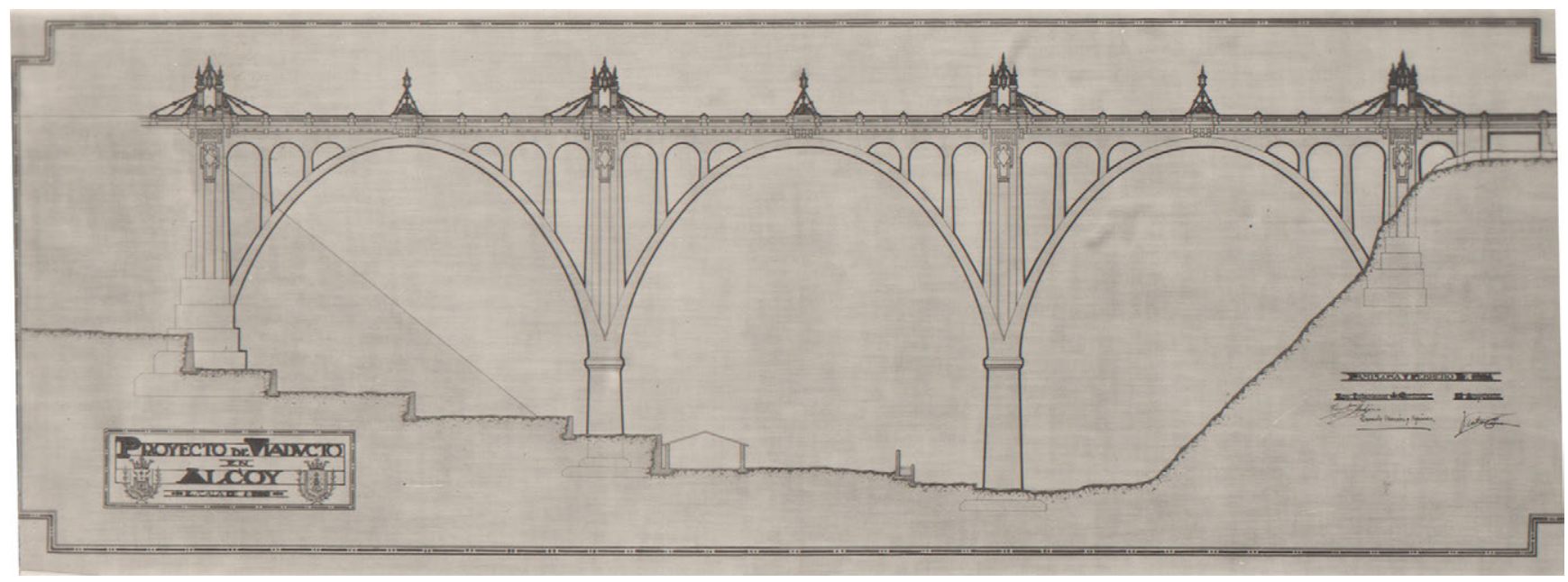

Figura 4. Alzado proyecto de febrero 1924 (fotografía del Archivo Municipal de Alcoy). 
de la empresa de elementos prefabricados de hormigón para conductos de ventilación SHUNT, S. A. Murió en Madrid el 7 de abril de 1970 .

\section{EL ARQUITECTO}

Carmelo Monzón y Víctor Eusa eran dos jóvenes impetuosos con un historial académico muy brillante, con ansias de materializarlo en alguna empresa digna, pero también con una posición progresista hacia lo nuevo. Una vez más, como tantas veces ocurre en la historia, esta casualidad condujo a la producción de una obra decididamente moderna que de otro modo no hubiera podido darse. La oportunidad del puente en Alcoy será un reto para ellos, un desafío bien colocado desde una empresa constructora que los amparará y aprovechará sus energías. No obstante, desde la arquitectura, el aporte moderno del puente de San Jorge de Alcoy corresponde fundamentalmente a la propia personalidad de este arquitecto y a su gusto, en este caso, como profesional del diseño de las formas.

Víctor Eusa nació en Pamplona el 6 de marzo de 1894 en la calle Estafeta, en la casa familiar en la que habría de vivir y trabajar hasta los ochenta años. Dejó la actividad profesional en 1979 y murió en Pamplona en 1990. Fue arquitecto titulado por la Escuela de Arquitectura de Madrid en 1920 y también fue Premio Extraordinario de Fin de Carrera. Fue uno de los arquitectos más brillantes de su generación y demostró durante sus estudios una asombrosa capacidad plástica y compositiva, con una habilidad especial para aplicar el lenguaje establecido en sus justos términos en todo su trabajo (9).

En la Escuela de Arquitectura hubo dos maestros que influyeron fundamentalmente en la formación de Víctor Eusa y que tuvieron una importancia decisiva en todas las generaciones de arquitectos de esa época: por un lado, Modesto López Otero, representante de la línea ortodoxa del academicismo con el que Eusa adquiriría una formación clásica, en la tradición Beaux Arts, con todo el rigor que ello suponía en el manejo del dibujo, en la reflexión a través de él con una destreza impecable, con un gran conocimiento de las aplicaciones del «tipo», con el sistema de composición basado en la jerarquización y con la generación del carácter del edificio a través de la cuidada aplicación del ornamento; por el otro lado, Teodoro de Anasagasti, representante de la apertura hacia los nuevos movimientos artísticos del cambio de siglo, sobre todo en lo que se refiere a la tendencia de la Sezession vienesa, maestro del que Eusa sería el alumno más distinguido.

Desde los tiempos de estudiante en la Escuela de Madrid, la curiosidad por el conocimiento de lo que ocurría en el mundo de la arquitectura a través de las revistas internacionales y de las publicaciones fue una constante de su trabajo. No hay que dejar de considerar que, ya entonces, la Biblioteca de la Escuela de Arquitectura de Madrid disponía de fondos muy amplios a disposición de los estudiantes y que las discusiones sobre las tendencias y movimientos eran comunes entre ellos. Así pues, Víctor Eusa conocía el material historiográfico que se producía en la Europa de aquellos tiempos con una voluntad muy decidida. Por lo tanto, no es de extrañar que supusieran un gran atractivo las publicaciones europeas de las obras de Frank Lloyd Wright, de Eric Mendelsohn, y de tantos otros arquitectos.

Inmediatamente después de finalizar los estudios de arquitectura ganó el concurso del casino del Gran Kursaal de San
Sebastián, al que se presentó con un proyecto de un edificio "en "estilo francés" conforme a las exigencias de la época» (10).

Después de los viajes ya todo se centrará en Pamplona, reuniendo la tradición académica y las tendencias modernas, para crear progresivamente un lenguaje personal. Se trata de un arquitecto muy prolífico, pero casi exclusivo y aislado en la ciudad de Pamplona y su entorno más próximo, en donde desarrollará cerca de un millar de obras y proyectos. Por tanto, será muy conocido en los círculos de los profesionales de la construcción de la ciudad. En Pamplona se posicionará y adquirirá una gran experiencia proyectual y constructiva, si acaso cerrada, pero vivirá de la rentabilidad de un lenguaje y un modo de componer propio, cimentado entre los signos y elementos del lenguaje moderno aunque sometido a un modo de proyectar clásico.

\section{UN PUENTE DE HORMIGÓN ARMADO VISTO}

Según la memoria del proyecto, la decisión de proyectar un puente de hormigón armado y no hacerlo de fábrica o metálico se basó en los criterios establecidos en las colecciones de modelos de puentes que de manera oficial se estaban redactando por figuras eminentes de la ingeniería nacional. Se trataba de Juan Manuel de Zafra, Domingo Mendizábal y José Eugenio Ribera, con la colaboración de Alfonso Peña Boeuf según se desprende de las ediciones posteriores de estas colecciones (11). De estos criterios surge la elección de proyectar inicialmente un puente con tres vanos que corresponde al mínimo de arcos con rebajamiento $1 / 2$ cuyo resultado final será un puente de tres arcos de $46 \mathrm{~m}$ de luz conformados con dos bóvedas gemelas con directrices de forma parabólica de cuarto grado (1). Después, el puente de San Jorge de Alcoy será citado por Ribera como ejemplo de los viaductos de hormigón armado en el tomo final de su publicación en donde se incluían estas colecciones de modelos de puentes (12).

El hormigón armado haría posible desechar los robustos modelos oficiales publicados muy condicionados por las alturas, anchuras y las longitudes de servicio basados en la herencia de los puentes de gravedad, pero también rechazar la experimentada técnica de los puentes metálicos, que aunque más audaces suponen una larga tarea de mantenimiento. Por entonces, para las luces y las alturas demandadas, el uso del hormigón armado ofrecía ventajas económicas dignas de consideración.

Tal y como ya era costumbre en muchos de los puentes urbanos, desde el concurso del puente de María Cristina en San Sebastián, los proyectistas cuentan con un profesional de la arquitectura para las cuestiones del embellecimiento y lo más importante: «... ha colaborado en este proyecto un Arquitecto no limitándose dicha colaboración a una simple decoración después de resuelta la parte de ingeniería sino que por el contrario aquella se ha dejado sentir desde el primer momento con lo cual el cálculo y la estética van íntimamente unidas evitándose así reformas enojosas que ante las exigencias arquitectónicas hubiesen sido hechas después» (1). Toda una declaración de principios sobre el modo de trabajar en un proyecto entre profesionales del mundo de las estructuras y de la arquitectura, entendiendo esta disciplina aquí como la experiencia en el control de la forma tendente hacia la belleza y la integración en la ciudad sin menoscabo de la función y de la estabilidad. 


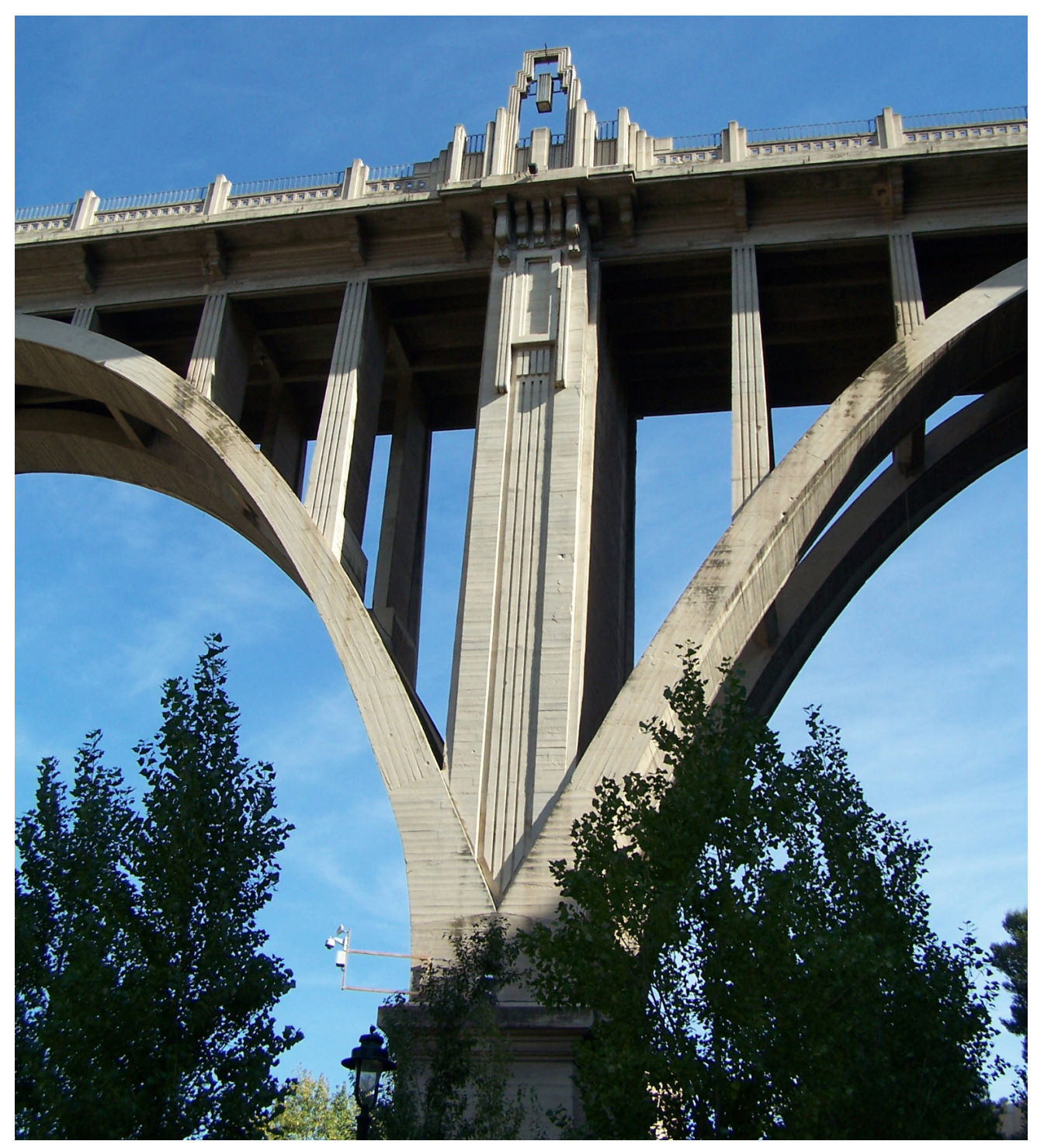

Figura 5. Pila central entre dos arcos (fotografía de la colección del autor).

Como rasgo importante del acercamiento a las tendencias de la modernidad, que se observará en la trayectoria de los proyectistas y que se pone de manifiesto ya en la propia Memoria de este proyecto, está su idea de la posición de los detalles ornamentales y su coherencia con el material con el que se va a construir el puente: «En cuanto a la ornamentación se ha tenido en cuenta los fines de las obras y el material de que se ejecuta: lo primero nos ha inducido a tratar la parte alta con más riqueza ornamental que la inferior ya que aquella formará parte de una vía urbana y esta se desarrolla en los terrenos próximos al río.» (1). La consideración de la parte alta como formando parte de un vía urbana, como una «calle» en definitiva, es crucial para entender la evolución hasta el resultado final de los cerramientos defensivos del Puente desde el interior del mismo. Ésta es una visión muy arquitectónica propia de una profesión sensible hacia las características de los espacios urbanos.

También con respecto al hormigón armado como material principal de la construcción del puente hay una tendencia decidida: "Siguiendo siempre con la idea de buscar formas propias a la estructura de hormigón armado hemos respetado para su decoración y ornamentación las líneas puramente constructivas lo que nos ha llevado a tratar todo con gran ligereza procurando acentuar en un máximo grado las líneas verticales dominantes en este proyecto: con ello hemos conseguido evitar el contraste grande que hubieran producido las pilas por su masa con relación a las demás partes componentes» (1). Por lo tanto, aquí se observan las intenciones al utilizar y dominar ciertos recursos formales, siempre con líneas puramente constructivas, para obtener un resultado que proporcione gran ligereza a unos elementos que de por sí resultarían muy robustos.

Pero también hay un gran interés en que el perfil del puente desde su visión lejana tenga una lectura de objeto compuesto en su totalidad con estos mismos criterios: «Estas mismas pilas, como elementos principales, las hemos acentuado en su parte superior con los motivos ornamentales íntimamente unidos a ellas y con estructura pura y simple de hormigón armado habiendo empleado como elemento auxiliar el hierro ya que su relación con el hormigón no puede ser más íntima y habiéndosele tratado con la mayor sencillez puesto que ese debe ser la impresión dominante de una obra de este género. Era preciso asimismo debido a la gran longitud de este viaducto evitar la monotonía el cansancio que hubieran producido inevitablemente la gran línea horizontal superior, lo que hemos conseguido al acentuar las pilas con los motivos 
ornamentales que hemos citado, y el punto más alto de los arcos con otros motivos menos importantes que aquellos» (1). Probablemente estas apreciaciones constituyen el único testimonio escrito de las intenciones de Víctor Eusa en su intervención como arquitecto en un trabajo como éste. Son argumentos sobre morfología y sobre la composición que normalmente son extraños a la ingeniería, al menos en los tiempos actuales, aunque también es cierto que entonces había ingenieros con una sensibilidad estética muy educada en estos menesteres.

Y como conclusión de estas tendencias fijadas en la Memoria del Proyecto: "No se ha hecho un solo despiezo, no se ha variado ni un solo plano de los exigidos por el cálculo, por entender que es preciso avanzar buscando las nuevas formas que exige este material y para ello es necesario antes que todo subordinar a las condiciones del mismo sin temer que en un principio puedan causar extrañeza estas innovaciones ante la rutina de las casas conocidas, respondiendo siempre a la lógica que deben guiar todas nuestras concepciones."

No conviene perder de vista que es la empresa constructora Erroz y San Martín quien se hace cargo de la aventura de la construcción del puente. La propia empresa busca a los redactores del proyecto, pero también influirá en algunas decisiones que le interesan legítimamente. Es muy probable que la decisión sobre la elección del sistema general de la construcción en hormigón armado fuese compartida y acordada entre proyectistas y constructores, no hay que olvidar que Erroz y San
Martín tiene una matriz como empresa de carpintería adaptada a la construcción, por lo tanto podemos considerarla como una constructora especialista en encofrados y cimbras.

El proyecto encargado tuvo fijadas las condiciones de alineaciones y rasantes que recogía el Proyecto de Ensanche y Rectificación de la Ciudad de Alcoy, redactado por Enrique Vilaplana y Teodoro Balanciart en 1878. Este Plan de Ensanche preveía un puente metálico de viga continua, acorde con la tecnología de la época, que salía del centro de la ciudad con un tablero horizontal y que desembocaba al otro lado sobre una avenida formada por varios arcos de fábrica de gravedad que se ajustaban a la llegada final con una rasante inclinada. Por esta circunstancia el proyecto inicial del puente de San Jorge presenta un tablero horizontal sobre un sistema de tres arcos parabólicos que coincide con el desarrollo del puente metálico del proyecto de ensanche de la ciudad. El tramo de la avenida inclinada del proyecto del ensanche queda fuera del encargo y es sustituido por un descomunal terraplén que gestionaría directamente el Ayuntamiento de Alcoy por expreso empeño suyo. Tradicionalmente en la ciudad el negocio de los vertederos de tierras y escombros era muy común y extendido en aquellos tiempos.

Así pues, el proyecto presentado solamente contempla un puente formado por tres vanos compuestos por tres grandes parejas de arcos parabólicos y un tablero horizontal. El tablero se descarga sobre los arcos mediante tabiques o pilastrillas

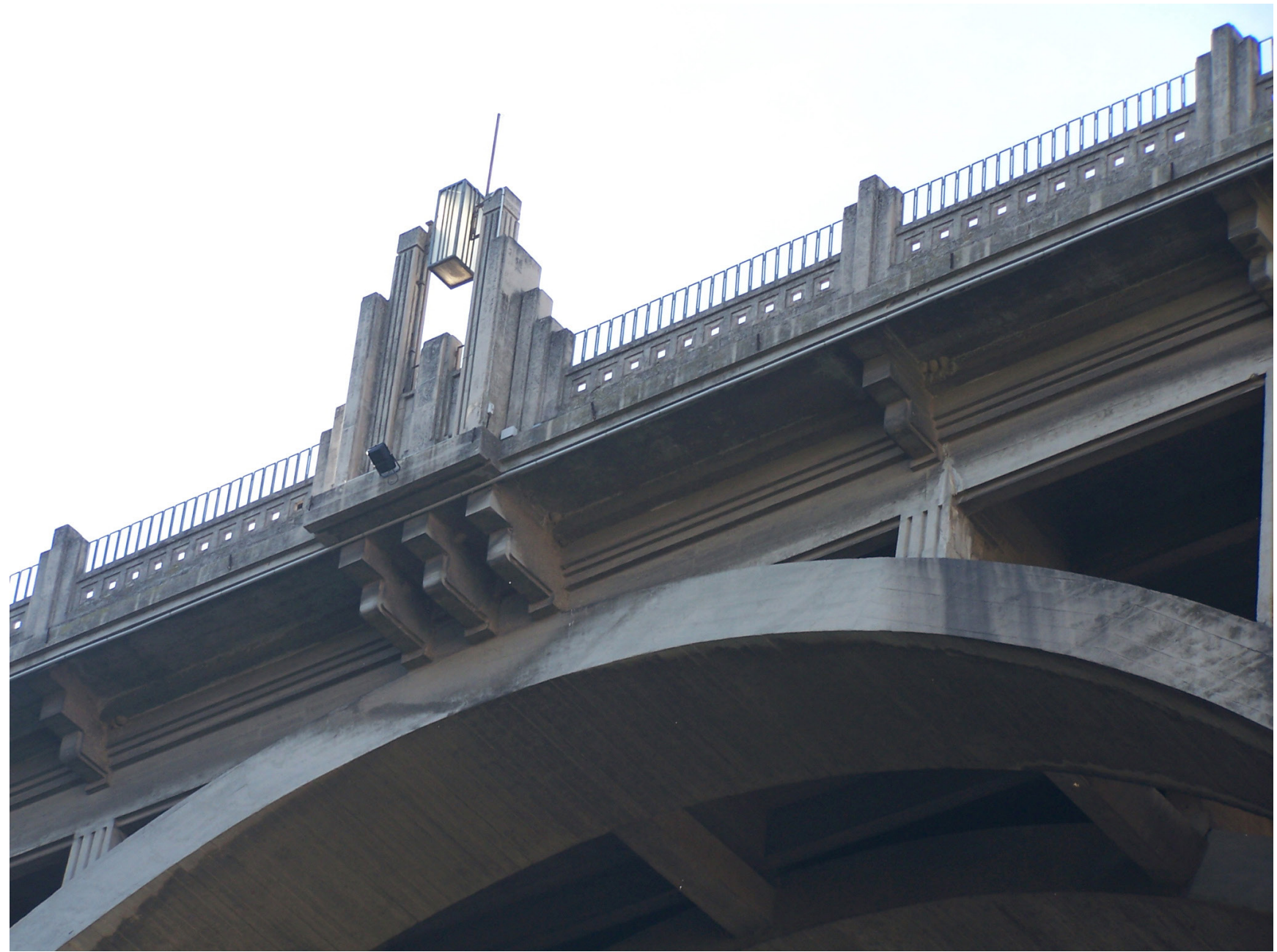

Figura 6. Columna decorativa sobre clave de los arcos (fotografía de la colección del autor). 
que se rematan con arcos de medio punto y en el alzado se observan perfectamente los detalles ornamentales previstos (Figura 4). Las pilas del puente, que tienen una masa considerable, son tratadas por el arquitecto con determinación colocando estrías y elementos decorativos que ajusten su esbeltez a las demandas de la visión lejana, bien organizados bajo la medida de la composición de las ménsulas. Aquí, los elementos de la composición tienen rasgos formales más avanzados, simples y geométricos característicos del lenguaje Art Déco, aunque están compuestos de una manera todavía clásica. En cambio, los elementos situados sobre el tablero, aquellos que quedan en contacto más directo con los usuarios del puente, las columnas decorativas que albergarán la iluminación y las barandillas, responden a un lenguaje ecléctico y tradicional a base de guirnaldas y balaustres que desvelan una cierta preocupación por la aceptación del diseño por parte de los usuarios del puente. Podríamos decir que en este proyecto inicial y con respecto a las decisiones del arquitecto Eusa, vencieron las posiciones de las tendencias del regionalismo tradicional que representaría López Otero en la escuela de arquitectura frente a aquellas más vanguardistas de Teodoro Anasagasti.

Curiosamente, nada más contemplar el puente construido se observa claramente que el puente proyectado era diferente (Figura 2).

\section{EL DESARROLLO DE LA OBRA}

Desde febrero de 1924, fecha que data la redacción del proyecto inicial, hasta el comienzo de la obra de construcción del puente el 27 de noviembre de 1925 el proyecto recorrió los trámites de la supervisión de la administración pública y aquellos relativos a la contratación de la obra. Pero también durante ese período los proyectistas van acercando a la realidad aquello proyectado desde dos cuestiones importantes:

1. La cuestión del terraplén previsto en el estribo izquierdo del puente había generado desde el principio grandes dudas en la mente de Carmelo Monzón desde el punto de vista estructural y constructivo -dadas las grandes dimensiones del mismo- pero también desde el punto de vista funcional. Estas dudas se materializan en una modificación del proyecto cuyos planos vienen fechados el 20 de diciembre de 1925, prácticamente un mes después del inicio de la obra, en los que el terraplén se reduce añadiendo al puente una ampliación formada por dos vanos de tablero recto, esta vez con la rasante inclinada, sobre pilastras manteniendo el mismo tipo de detalles decorativos del proyecto inicial. Esta solución se presentó oficialmente el 23 de marzo de 1926 y la solución que se aprobó finalmente a finales de agosto del mismo año ya contemplaba una nueva ampliación de dos vanos rectos más que aquella presentada anteriormente. Por lo tanto, desde el inicio de la obra se trabajó afanosamente en la modificación del proyecto (Figura 3).

2. Al mismo tiempo que todo esto va ocurriendo, el arquitecto Víctor Eusa continuó su trayectoria profesional evolucionando hacia posiciones más avanzadas. Seguramente fue determinante para él la participación y la asistencia a la Exposición Universal de las Artes Decorativas de París que cerró sus puertas en octubre de 1925. Esta exposición contribuyó entre otras cosas a la aceptación de la modernidad por la sociedad y, probablemente, le dio el coraje suficiente a Víctor Eusa para modificar los elementos decorativos de corte regionalista y ecléctico hacia las posiciones del lenguaje Art Déco que ahora podemos disfrutar (Figura 5). Estos cambios suponían una modificación sustancial en las formas de los detalles de las barandillas y de los remates de las pilas y pilastrillas que debieron estar listos ya en la construcción de los arranques de los tabiques dispuestos sobre los arcos y las pilas centrales, entre febrero y abril de 1926 (Figura 6). El plano del alzado del puente redactado por Eusa que realmente expresa lo que fue construido tiene fecha del primero de junio de 1926.

En noviembre de 1926 la Revista de Obras Públicas (7), de la mano de Alfonso Peña Boeuf, da noticia del descimbramiento del primer arco del puente. Este ingeniero es testigo de este acto y en el relato cuenta que los arcos extremos

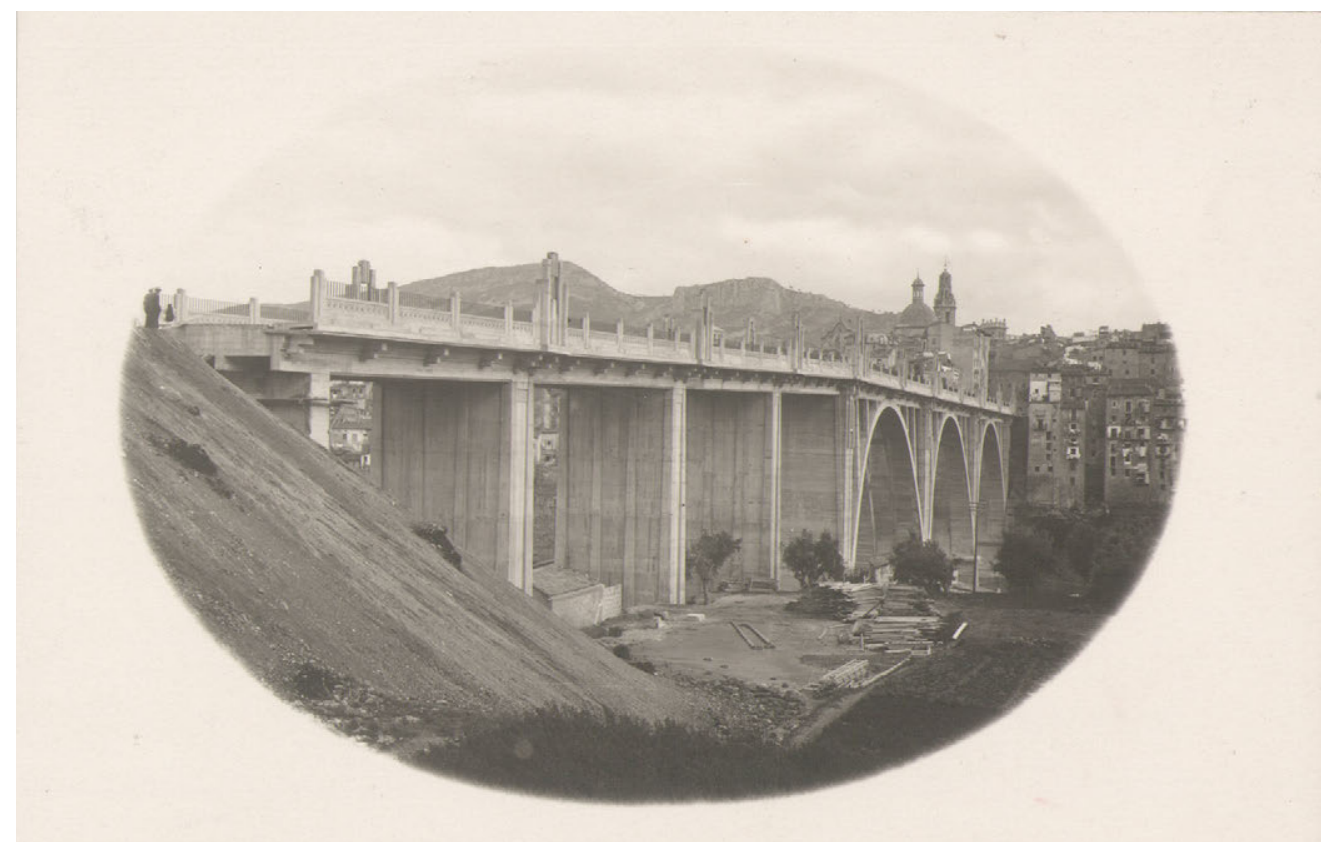

Figura 7. Vista histórica desde el estribo izquierdo con terraplén de acceso sin terminar (fotografía del Archivo Municipal de Alcoy). 
se encuentran hormigonados y se está en proceso de vertido del hormigón en el arco central. En la sesión de la Comisión Permanente del Ayuntamiento del 2 de noviembre de 1927 se aprueba la cesión de la explotación de la cantera que utilizaba la empresa Erroz y San Martín para fabricar el árido para el hormigón del puente. Esto significa que en octubre de 1927 las labores de fabricación de hormigón en la obra del puente han terminado. Pero no sabemos cuándo se terminaron las operaciones de construcción efectivamente. Desde luego no hay queja oficial alguna sobre la finalización de la obra prevista para el 1 de julio de 1927. El terraplén de la parte izquierda no estaba listo todavía para hacer practicable el puente (Figura 7). El 23 de marzo de 1928 se levanta un acta sobre la realización de las pruebas de carga del puente. Con los resultados de las pruebas de carga, se hizo entrega del puente por la empresa constructora al Ayuntamiento. Carmelo Monzón visitó e inspeccionó la obra para proceder al levantamiento del Acta de Recepción Definitiva de las obras entre el 25 y el 30 de octubre de 1928.

\section{CONCLUSIÓN}

El gran esfuerzo de coordinación de las voluntades del ingeniero y del arquitecto, el tiempo de su dedicación a esta obra, su trabajo, buena voluntad y honradez pueden vislumbrarse si se tiene en cuenta el alcance y el valor de las modificacio- nes que este puente tuvo. El ajuste de todas las medidas de cada uno de sus elementos incluidos en un sistema general completamente decidido que ahora podemos contemplar, los cálculos entre dos sistemas estructurales diferentes situados en la vanguardia de la época, los detalles constructivos de los complicados encofrados necesarios sólo pueden resolverse con éxito por medio de una estrecha colaboración entre ingeniería, arquitectura y empresa constructora, con oficio y reflexión responsable. No obstante, en este caso, todo este esfuerzo vino promovido por el empeño decidido en manifestar técnica y estéticamente el espíritu de los tiempos que corrían, con el propósito de conseguir la belleza a través del diálogo interno y de las reflexiones entre los proyectistas que escaparon en todo momento de la banalidad, de la ligereza, de la improvisación y de la irresponsabilidad cuyo resultado es la fealdad que acompaña a muchas obras.

Las obras son el fruto de los hombres que trabajaron en ellas, hombres cuyo trabajo acaba olvidándose. El puente de San Jorge de Alcoy, olvidado también por los intereses de otros tiempos que vinieron después de su construcción, muestra las tradiciones constructivas que todavía hoy pueden reavivarse a través del coraje de su nada orgullosa presencia. Sirva, pues, este testimonio para conmemorar la dignidad de sus noventa años y para rescatar del olvido a los hombres que lo construyeron.

\section{REFERENCIAS}

(1) Archivo Municipal de Alcoy, expediente signatura 3641-II.

(2) Marcos, I., San José, J. T., Cuadrado, J. (2014). Las patentes en la introducción del hormigón armado en España: caso de estudio de la Alhóndiga de Bilbao. Informes de la Construcción, 66(534): eo24, doi: http://dx.doi.org/10.3989/ ic.13.032.

(3) De Alzola, P., de Churruca, E. et al. (1904). Puente sobre el río Urumea. Concurso de proyectos abierto por el Excmo. Ayuntamiento de San Sebastián. Informe del Jurado. Revista de Obras Públicas, Año 52, tomo I: 110-116.

(4) Ribera, J. (1908).Los puentes modernos. Revista de Obras Públicas, Año 56, tomo 1: 349-352.

(5) Ribera, J. (1924). La Construcción y la Arquitectura en la Exposición de Londres. Revista de Obras Públicas, Año 72 , tomo 1: 349-352.

(6) Rovira, T. (2010). Estructura y forma, compañeros de viaje de Owen Williams de la ingeniería a la Arquitectura. Proyecto, Progreso, Arquitectura, «Viajes y traslaciones» (3): 94-109.

(7) Peña, A. (1927). Descimbramiento del Viaducto de Alcoy. Revista de Obras Públicas, Año 75, tomo 1: 3-4.

(8) Grandes, A. (2010). Inés y la alegría. Episodios de una guerra interminable, p. 22, Barcelona: Tusquets Editores.

(9) Tabuenca, F. (1989). Presentación. En Tabuenca, F. (Ed.), Víctor Eusa Arquitecto (p. 7). Pamplona: Exmo. Ayuntamiento de Pamplona, COAV-N e Institución Príncipe de Viana.

(10) Linazasoro, J. I. (1973). Víctor Eusa. Nueva Forma: Arquitectura, Urbanismo, Diseño, Medio Ambiente y Arte (9o91): 2.

(11) Ribera, J. (1929). Puentes de fábrica y hormigón armado, tomo III, p. 99-100. Madrid: Talleres Gráficos Herrera.

(12) Ribera, J. (1932). Puentes de fábrica y hormigón armado, tomo IV, p. 247. Madrid: Talleres Gráficos Herrera. 